Published in PerDis '19 Proceedings of the 8th ACM International Symposium on Pervasive Displays. Palermo, Italy - June 12 - 14, 2019. New York, NY, USA: ACM, 2019. ISBN:978-1-4503-6751-6.

https://doi.org/10.1145/3321335.3324931

\title{
Acceptance and Perceptions of Interactive Location-Tracking Displays
}

\author{
Ville Mäkelä* \\ ville.maekelae@ifi.lmu.de \\ LMU Munich \\ Germany
}

\author{
Juhani Linna \\ juhani.linna@tuni.fi \\ Tampere University \\ Finland
}

\author{
Tuuli Keskinen \\ tuuli.keskinen@tuni.fi \\ Tampere University \\ Finland
}

\author{
Jaakko Hakulinen \\ jaakko.hakulinen@tuni.fi \\ Tampere University \\ Finland
}

\begin{abstract}
Tracking the location of people and their mobile devices creates opportunities for new and exciting ways of interacting with public technology. For instance, users can transfer content from public displays to their mobile device without touching it, because location tracking allows automatic recognition of the target device. However, many uncertainties remain regarding how users feel about interactive displays that track them and their mobile devices, and whether their experiences vary based on the setting. To close this research gap, we conducted a 24-participant user study. Our results suggest that users are largely willing - even excited - to adopt novel location-tracking systems. However, users expect control over when and where they are tracked, and want the system to be transparent about its ownership and data collection. Moreover, the deployment setting plays a much bigger role on people's willingness to use interactive displays when location tracking is involved.
\end{abstract}

\section{CCS CONCEPTS}

- Human-centered computing $\rightarrow$ Human computer interaction (HCI); Ubiquitous and mobile computing; • Security and privacy $\rightarrow$ Social aspects of security and privacy.

\section{KEYWORDS}

public displays, location tracking, acceptance, perceptions, privacy, trust, location-based services, mobile devices, ubiquitous computing

\author{
Markku Turunen \\ markku.turunen@tuni.fi \\ Tampere University \\ Finland
}

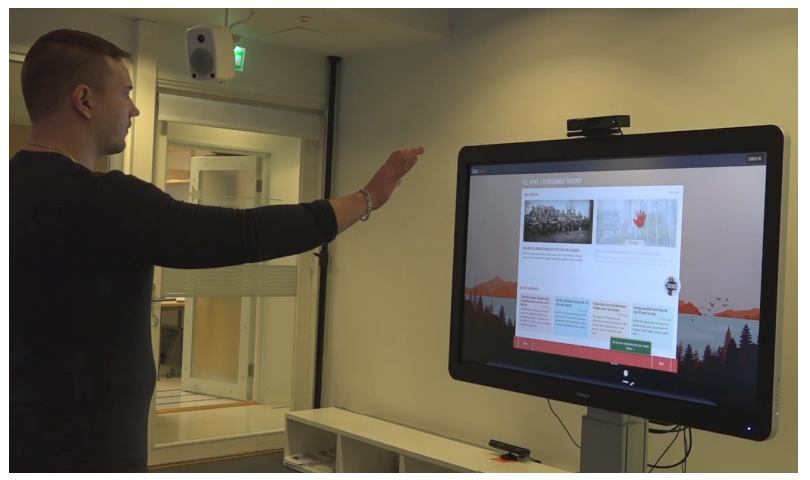

Figure 1: The SimSense system. SimSense utilizes location tracking, of both the user and the user's mobile device, to automatically pair mobile devices with their owners. Consequently, users can pull content from the display to their mobile device from a distance, without ever touching the mobile device.

\section{INTRODUCTION}

Creative utilization of technology has enabled advanced interactions for public displays, particularly through the use of location tracking [23, 29, 34, 35]. A prime example of such is the SimSense system (Figure 1). SimSense ${ }^{1}$ uses location tracking to automatically pair users with their mobile devices and utilizes mid-air gestures to allow content retrieval from a distance. Therefore, users never need to take out their mobile device from a bag or pocket and can interact with mid-air gestures without the need to walk up to the display. Such seamless interactions are efficient and provide a highly positive user experience [23,24], and are important in shaping future ubiquitous environments to support blended and natural interactions $[2,30]$.

Despite these advances, prior research identifies many factors that hinder the wide adoption of - and interaction with - new technologies especially in public spaces. For example, we already know that users may choose to not interact with technology due to lack of attraction or motivation [21], fear of embarrassment [7], uncertainties regarding the interaction [26], or desire to avoid disclosure of the content they interact with [32]. However, novel

${ }^{1}$ https://www.youtube.com/watch?v=RkpjCsNBu3U 
interactive systems introduce many more factors that may influence people's willingness to interact with them. Primarily, implications from tracking the location of people and mobile devices in public, and pairing people with their devices automatically, are unknown. We also lack a clear understanding of how much the deployment setting (for example, whether it is a shopping mall or a library) influences the users' willingness to interact with location-tracking systems.

Understanding the implications of location tracking and automatic pairing in public spaces has significant influence on the design, deployment, and ultimately the success of future interactive systems. Therefore, we invited 24 participants to interact with the SimSense system and provide feedback, particularly regarding their concerns about location tracking and the use of novel interactive systems in various settings. Accordingly, our primary research questions were:

- RQ1: What are the users' concerns of, and requirements for, interactive displays that track the location of people and personal devices?

- RQ2: How does the deployment setting influence the willingness to use interactive location-tracking systems?

Our findings suggest that users are largely willing - and even eager - to adopt novel location-tracking systems, provided that the system is transparent about its data collection and ownership and allows users to set their tracking preferences. No major concerns or deal breakers were found. However, introducing location-tracking features to an interactive display greatly emphasizes the influence of the deployment setting on people's willingness to use them. Calm and/or professional environments, such as museums, libraries, office workspaces, and university campuses are preferred over less organized settings, such as city centers and shopping malls.

\section{RELATED WORK}

In this paper, we use SimSense [23, 24] as an example of novel interactive systems that track the location of both people and personal devices. Other novel tracking solutions that enable automatic association of mobile devices with people have also been proposed $[29,34]$. Of relevance is also the work by Wu et al. [35], who do not do user-device pairing per se, but nonetheless are able to track people and their actions in the space, including the presence and use of mobile devices. However, none of the aforementioned works deal with the concerns that might accompany these technologies, and to our knowledge, no acceptance studies have been conducted in this context.

The issues of trust and privacy have previously been studied in the context of personalized public displays, for example, based on mobile devices that come into the vicinity of the display [ 10 , 12]. These systems are likely to contain similar implications. It is important to note, though, that the approaches we evaluate in this paper go further, for instance, by making assumptions about device ownership, and by allowing content to be sent to a personal device that is not directly manipulated by its owner.

In a broader spectrum, there is a plethora of studies that provide insight into the perceptions and acceptance of location tracking, location sharing, and privacy [3]. Interestingly, Liu et al. [18] point out in their recent literature review that people's views on location privacy have changed over time: early studies (before 2010) show little concern for location privacy, but more recent studies show otherwise - this change in itself serves as further motivation for this study.

Existing research has mostly studied the implications of location privacy in mobile computing contexts. Most notably, users' willingness to share their location varies with the place and social context they are in [1], as well as who is requesting or getting the information [11,17]. Similarly, Barkhuus and Dey [5] found that users were more concerned about location privacy when the location was tracked by other parties, as opposed to only the device's awareness of its own location. Our study presented in this paper concerns a public display being aware of people and private devices and is therefore fundamentally different from the aforementioned studies. Nonetheless, it is reasonable to consider that similar implications might be found in the context of location-tracking interactive displays.

Want et al. [33] investigated a location system at workplaces using badges and found that even though there were initially a multitude of concerns about privacy, they were largely overcome among working professionals after the benefits of the system became apparent to them, and after they became more trusting of the fact that the collected data was not abused. However, there was a strong desire for the employees to be able to opt out of the system whenever they wanted.

Our work also links to the so-called dark patterns, which were originally presented by Brignull [6] as parts of a user interface that do not have the users' interests in mind and that are crafted to trick users into doing things they might not be aware of. Greenberg et al. [13] foresaw that dark patterns might be a considerable problem in proxemic sensing systems that track and determine spatial relationships between people and devices. Greenberg et al. define dark patterns broadly, and also include so-called anti-patterns. Antipatterns, as opposed to dark patterns, are unintentional, yet may lead to similar results.

Dark patterns and anti-patterns certainly play a role in the acceptance of interactive systems. If users think or fear that not everything the system does is to their benefit, it might significantly hinder the system's acceptance (regardless of whether the system actually does this). A particularly relevant dark pattern is called disguised data collection [13], which refers to the system gathering information and using it for purposes the user is not aware of. We will reflect our results on the various dark and anti-patterns towards the end of our paper, when we discuss our results.

In summary, the acceptance, concerns and privacy implications regarding location tracking and location sharing is a widely researched topic. However, to our knowledge, there are no studies that explicitly focus on this topic in the context of interactive location-tracking systems. While some studies touch on this subject, they focus on different perspectives, such as personalization [10] and technical solutions [12]. Most other studies have focused on the mobile context, for example, whether users accept that mobile applications track the device's location, or how willing users are to share their location with others, such as their friends, bosses, and spouses. The fundamental difference, then, is that interactive location-tracking displays track the users and their mobile devices to enable interactions with a public device (the display), available to 
anyone, and available to be observed by anyone. Understanding the potential concerns and requirements that this creates is important for the success of novel interactive systems, especially because their success is already threatened by hesitance for interacting in public (e.g., [7, 26]) as well as external, often uncontrolled forces [25].

\section{SIMSENSE}

SimSense is a smart space system that enables seamless transferring of content from public displays to personal mobile devices (Figure 1). SimSense tracks the location of users through a Kinect sensor, and the location of mobile devices using Bluetooth beacons. Users and mobile devices with matching locations are automatically paired by the system. Consequently, when a user interacts with the system and chooses content to be transferred, the system already knows which device to send the content to. For the automatic pairing and content transfer to work, a mobile application needs to be installed. Detailed descriptions of the user interfaces and the implementation of the system are provided in [23, 24].

Originally, SimSense utilized mid-air gestures, allowing interaction from a distance as people pass by the display [23], which resulted in a highly positive user experience. SimSense was later upgraded into a multimodal system, supporting interaction also with touch, gaze, and a combination of gaze and mid-air gestures [24].

\section{USER STUDY}

We recruited participants from our university's basic course Introduction to Computing. The course is mandatory in almost all study programs, and is offered also in the open university curricula. Roughly 1200 students take it every academic year, and the background and computer literacy of the students is highly varied. Participation in the study was voluntary; however, participants were offered a small number of exercise points as a reward. Exercise points contributed towards passing the course, but had no effect on the final g rade. Participants registered for the study by first filling out an online questionnaire, through which they provided basic background information as well as answered statements about location tracking and mobile device use.

This way, we recruited 24 participants ( 9 males, 14 females, 1 other). The recruited participants were $19-50$ years old $(M=28$, $\mathrm{SD}=8.9$ ). Almost all participants had at least some experience with gestural interfaces, as well as with interactive public displays. Participants were very mixed about how mobile applications collect their data or follow their location: some were concerned, some undecided, and some did not worry at all. Equally mixed results were received when we queried about the participants' willingness to use their own mobile devices in public.

In the study, participants were first given a short introduction to SimSense and its purpose. Then, a smartphone with the SimSense mobile application installed was handed to them, which they put in one of their pockets. Participants were then asked to explore the system and transfer several items from the screen into the mobile phone in their pocket. Due to the focus of the user study, this phase was rather free-form. The primary aim of this phase was to ensure that the participant understood the purpose of the system, knew how interaction with it works, and understood that the system tracks the location of both the user and the mobile device to enable the automatic user-mobile pairing. Detailed information - technical or otherwise - about how exactly the system works was not given unless the participants themselves asked questions. We rationalized that this way, participants would better represent real-world users: it is reasonable to assume that most users would not know about the fine details of a system's implementation or know how a system collects data and how the data are handled.

After the interaction, the participant filled out an online questionnaire containing statements on a 7-point scale. First, the questionnaire included generic statements to which participants answered whether they agreed or disagreed. Second, they assessed individual functions of location-tracking systems with regards to how comfortable or uncomfortable they felt about them. Third, participants were asked to rate various locations based on how they would feel about a) interacting and b) being tracked in those locations. The presented locations - library, office workspace, city center, museum, swimming/sports hall, university campus, shopping mall, and bus stop - were chosen based on their popularity or potential as deployment locations for interactive displays, as well as their appearance in current literature, e.g., [8, 9, 14, 15, 19, 20, 22, 26-28, 31]. We provided a picture of each location to assist in imagining said locations and presented a few examples of transferable content that could potentially be available in each of them. For example, in the library, the examples of transferable content included indoor maps and electronic books. We rationalized that this way, participants would have a better understanding of the spectrum of location-tracking systems, so that they would not rely too much on their personal experience with SimSense in just one location. Moreover, as SimSense and the interaction method were novel for every participant, we wanted to give practical examples as starting points for the interaction experience, and the questionnaire and interview that followed.

Finally, the participants were interviewed to gain further insight into their concerns and to understand the reasoning behind their questionnaire answers. The interview took around 15-20 minutes, and the whole session lasted an average of 40 minutes.

\section{RESULTS}

In the following, we present our results divided into three main sections: (1) concerns and requirements, (2) the influence of the deployment setting, and (3) general feedback. For the analysis of the questionnaire data, we used non-parametric tests (Friedman's and further Wilcoxon signed-rank) because the data were of ordinal scale and not normally distributed.

\subsection{Concerns and Requirements}

In our study, we used SimSense as an example of a novel interactive system. In the case of SimSense, location tracking is utilized to enable seamless transferring of content from public displays to personal mobile devices. Although our study is not strictly about content transfer systems, we additionally included some contentrelated statements, which also served as a baseline. For example, prior work already suggests that people might be hesitant towards disclosing what type of content they are interested in [32]. 


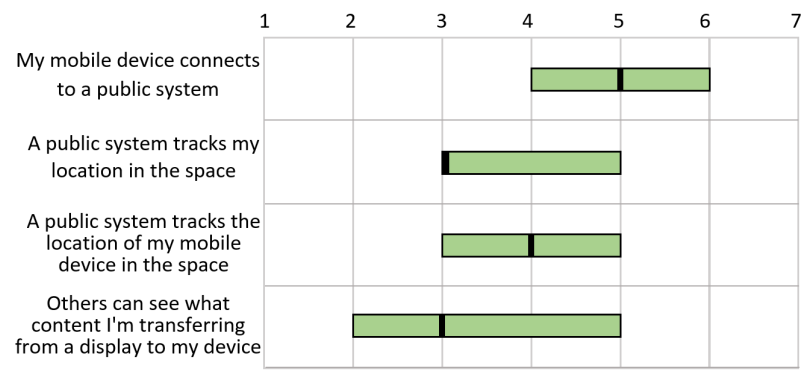

Figure 2: Participants' assessments on how comfortable they would be with the listed actions $(1=$ uncomfortable, $7=$ comfortable). The boxes represent the interquartile ranges and the thick, black lines represent the medians.

As such, we identified four unique features of SimSense that might affect people's willingness to use it: 1) users' personal devices connect to a public system, 2) the system tracks the location of users, 3) the system tracks the location of personal devices, and 4) the act of transferring content is visible to bystanders. Hence, we asked participants to rate the four characteristics with regards to how comfortable they felt about them (Figure 2).

Friedman's test showed statistically significant differences between the ratings of the four features $(\chi 2(3)=20.32, p<0.001)$ Participants were not bothered by having their mobile device connect to a public system (Mdn $=5)$, and a Wilcoxon signed-rank test showed statistically significant differences between this and all the other features $(p<0.05)$. The other three features were rated lower, and there were no statistically significant differences between them. Median-wise, participants had a neutral opinion on the tracking of their mobile device $(\mathrm{Mdn}=4)$ but were somewhat concerned about themselves being tracked $(\mathrm{Mdn}=3)$ and others being able to see what content they interact with $(\mathrm{Mdn}=3)$.

In the interview, we asked about the participants' concerns and feelings with two steps. First, we asked what worries or remarks they had about the system. Then, if the participant did not mention any privacy or tracking -related issues, we asked them again more explicitly about whether they had such concerns. This follow-up question was presented to 14 participants (58\%), since they did not bring up privacy or tracking in any way in their initial answer.

A total of 11 participants (46\%) did not express any concerns about the system, most of whom explicitly stated that they had no concerns at all. For example, one participant explained:

"I can't really imagine being worried. There have been plenty of technology in the past about which people had irrational worries. And security-wise, I believe these things are built by capable teams who will make them safe." (Male, 24)

Those who were able to comment on the concern-related questions did not ultimately provide any remarks that were outright negative. Rather, the provided comments were pre-requisites for how such systems should behave in order for the respondents to use or keep using them, or statements of specific situations in which they would choose not to interact.

The most popular requirement was that the collected personal data must be respected ( 9 participants, $38 \%$ ). Respondents further explained that the collected data should not be used for any other

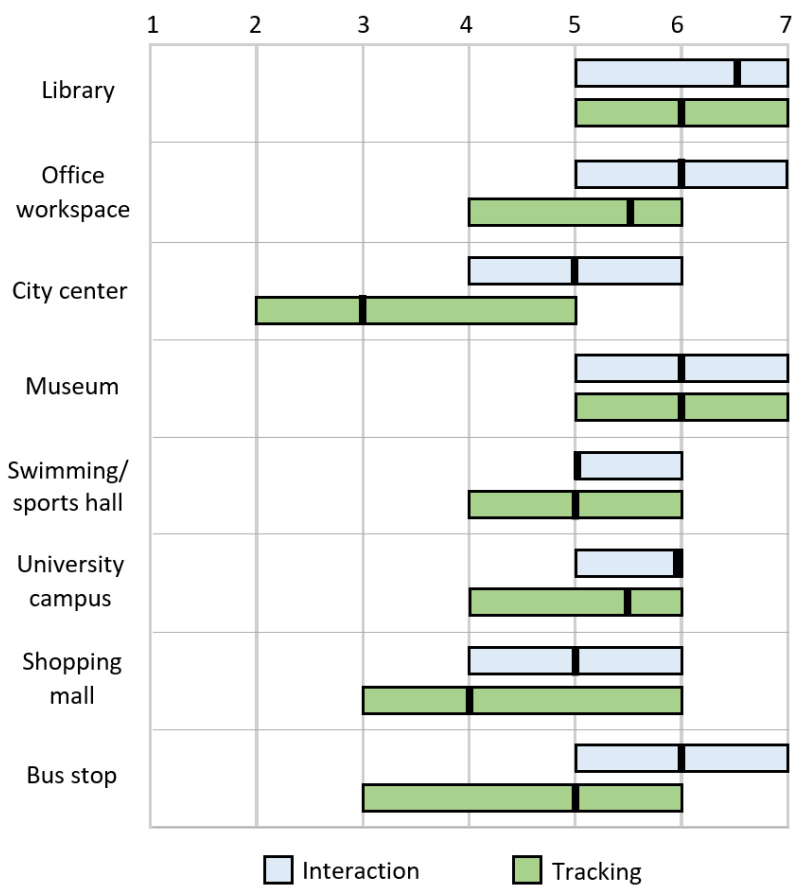

Figure 3: Participants' assessments on how comfortable they would be with a) interacting, and b) being tracked in the listed settings $(1=$ uncomfortable, $7=$ comfortable $)$.

purpose nor passed on to third parties, and that the system should only collect data that it needs to function. Several respondents continued by explaining that the system's (assumed) ownership would affect their attitudes towards the system, for example:

"It [concerns] would depend on who controls it [the system]. If it was some publicly run thing, I would not be bothered, but if it was run by some operator who might sell my information to third parties, it would be different." (Male, 34)

"[Willingness to use] depends on who manages and controls the system." (Male, 44$)$

Five participants (21\%) expressed that it is important for them to maintain control by being able to turn the tracking on and off whenever they please, or by setting preferences about where the tracking is enabled. This also linked to remarks about being fully aware of when the system is working and when it is not. For example, participants explained:

"At what point does the system stop tracking me? It would be nice to know clearly when it's in use and when it's not. I would want this to only track me in certain places. The boundaries would need to be clear, or I could set them myself, so that when I leave the area, the tracking stops." (Male, 23)

"It would be great if I could enable and disable the tracking from the app whenever I please." (Female, 23)

"Preferences should be available for setting when I want to be tracked and when I don't." (Male, 44) 


\subsection{The Influence of Setting}

Users assessed different settings based on how comfortable or uncomfortable they would be using interactive location-tracking displays in those settings (Figure 3). The assessment was separated to a) interaction, i.e., how comfortable they would be interacting, and b) tracking, i.e., how comfortable they would be with the system tracking the location of them and their mobile devices, at the specified place.

Generally, participants were more comfortable with interacting with a display than with having a display track them, as in six out of eight locations interaction was rated more positively, and in the remaining two they were rated equally.

Interaction in different public settings was overall rated rather positively (Mdn $=5-6.5)$. The highest rated setting was the library $(\mathrm{Mdn}=6.5)$, followed by office workspace, museum, university campus, and the bus stop ( $\mathrm{Mdn}=6$ ). City center, swimming/sports hall, and shopping mall received the lowest (but still positive) ratings $(\mathrm{Mdn}=5)$.

With comfortability towards tracking, more variance was observed. Still, the city center $(\mathrm{Mdn}=3)$ and the shopping mall (Mdn =4) again received the lowest ratings. Also following a similar trend, the most favorably rated settings were the library, museum, office workspace, and university campus ( $\mathrm{Mdn}=5.5-6)$, while swimming/sports hall and bus stop situated in the middle $(\mathrm{Mdn}=5)$.

We ran the Friedman's test to investigate the influence of the setting, and statistically significant differences were found in both the interaction category $(\chi 2(7)=26.03, p<0.001)$ and the tracking category $(\chi 2(7)=47.63, p<0.001)$. We leave out detailed pairwise comparisons because we do not intend to compare specific settings, but rather, assess the overall trends on what types of settings may be preferred over others and how much influence different settings have. As such, our interesting finding is that in the interaction category, a Wilcoxon signed-rank test showed statistically significant differences $(\mathrm{p}<0.05)$ only between 5 pairs of out the 28 possible pairs. However, in the tracking category, statistically significant differences $(p<0.05)$ were found between 15 pairs. This result suggests that the setting plays a much bigger role in the acceptance of interactive systems when location tracking is involved.

In the interview, users also made comments about the suitability of specific settings, and these comments seemed to suggest that many users reflected the trade-offs (location tracking) on how easily they could understand the system's benefit in that specific setting. In this way, calm and professional environments were also prominent. For example, several users explained that they would like to use such systems in a museum because there is a lot of useful information that they might want to interact with.

Finally, we inquired about which characteristics in the environment or the situation would affect the users' willingness to use interactive systems like the one they had just experienced. Half of the participants $(12,50 \%)$ reported that the presence of (a lot of) other people would likely make them uncomfortable. The most common explanations were that they would not want to gain unwanted attention, and they would not want others to see what kind of content they interact with, especially if they decide to transfer the content to their personal device. On a related note, six participants $(25 \%)$ stated that the type or sensitivity of the content would affect their willingness to use the system. Finally, five participants $(21 \%)$ reported that calm and organized settings or situations would be preferable. For example, two participants explained as follows:

"A safe and calm environment would be more comfortable. Also, in case there are problems, it would help if I knew there was a help desk or something where I could seek help." (Female, 31)

"I would not like hectic places where there's a lot of noise and movement. Places that are calm would work." (Female, 23)

\subsection{General Feedback}

Participants were seemingly positive about their experience. As also found in prior studies [23, 24], participants made comments about how futuristic and "cool" the interaction felt. They also provided various scenarios where they thought such systems would be useful. Some other comments from participants were:

"This is a cool idea. I'd like this to be available everywhere." (Male, 23)

"I've never heard of such possibilities before. This does make information retrieval easier." (Male, 23)

"I find constant use of a smartphone annoying. This would alleviate that because I would no longer need to take it out for certain things." (Female, 23)

"An interesting and sensible concept to make larger wholes smaller. Currently things like these would be done with QR codes, which are infuriating to use. It's great that it is being challenged in new ways." (Male, 33)

\section{DISCUSSION}

Our results suggest that users are largely accepting of, and excited about, interactive location-tracking systems, with some conditions. Generally, participants provided positive feedback and seemingly excited comments and were able to see the value that such systems can provide. This links to some earlier studies where people were found to accept location or information sharing if the received benefit was significant enough $[4,18,33]$.

\subsection{Requirements for Interactive Location-Tracking Systems}

Participants did not report any persistent issues with locationtracking systems that would make them reject such systems. However, we identified three requirements that future systems should consider:

Respect for private data. The most common requirement was that location-tracking systems should not collect or use data in ways that the user is not aware of, for example by selling the data to third parties. This links to the dark pattern disguised data collection, as predicted by Greenberg et al. [13]. However, it is notable that participants seemed to trust that their data would be respected in most cases, although some exceptions came up as discussed next.

Transparency about system ownership. Some participants reported that their willingness to use an interactive location-tracking system would depend on who controls and manages it. This links to the previous requirement about respecting private data, as users seemed to exhibit some distrust towards commercial companies that (they thought) might make use of the collected data or try to sell something to users. Participants seemed to be more trusting 
of systems that were run as a public service (e.g., by a city or a university). We believe that transparency about the ownership and purpose of such systems may foster their wider adoption.

User must remain in control. Participants reported that they would expect to be able to set the tracking on and off at will. Moreover, participants expressed general desire for being able to set preferences, such as in which types of settings tracking is enabled. These findings link particularly to Langheinrich's notion of choice and consent in privacy-aware systems [16]. A few participants also commented that they would like to be better aware of where the borders of the tracking areas are.

Overall, our findings suggest that people's a ttitudes towards interactive location-tracking systems are positive. The identified requirements largely follow the same trends as other location-based applications (e.g., $[5,11,17])$, and no comprehensive issues were found that would completely make users reject such systems. In fact, in our results we expected to find much more links to the dark patterns and anti-patterns [13], but they remained largely inconspicuous. It is important to note, though, that our study was not an investigation of the existence of these patterns, but rather, whether users' concerns link to them.

Finally, it should also be noted that our study of 24 participants, even if varied by age and educational background, is not necessarily a comprehensive representation of the target audience. Furthermore, the fact that this study did not uncover any major concerns about public location-tracking systems does not necessarily mean that there are no such concerns, or that we should not pay attention to them. What our study does, however, is it provides preliminary insights into the acceptance and perceptions of public locationtracking systems and sends a positive message to the research community that there is demand for, and willingness to use, novel location-tracking systems, if certain criteria are met.

\subsection{The Influence of Setting on Willingness to Interact}

One of our most interesting results is that users had much stronger preferences regarding different deployment settings when it came to location tracking than when it came to interaction. To clarify, the preferences did not change from one setting to another; instead, the existing preferences were amplified. This result suggests that an ideal deployment setting is even more critical for interactive location-tracking systems than it is for more conventional interactive systems. This should not be overlooked, because we already know from existing research that the setting has considerable influence even on traditional interactive technology such as public displays $[7,25,26]$.

Our results show a trend where calm and/or professional settings were preferred, such as libraries, museums, office workspaces, and university campuses. Settings that are open and potentially more crowded and hectic were rated lower, such as city centers and shopping malls. These results link with the results of Clinch et al. [10]: in the context of personalized public displays, users reported personalized displays more useful in semi-public locations as opposed to fully public locations, and similarly more useful in situations where they are lingering in the space as opposed to just passing through. In the context of more conventional public displays, Ojala et al. [26] found that leisure-related settings were more successful than serious settings; however, their displays contained multiplayer games (among other things), and thus attracted teens and children and fostered social interaction, whereas our study focused on fundamentally different issues. What remains unclear from our study, though, is how seamless interactions enabled by location tracking are affected by social factors, for instance, when multiple users are interacting simultaneously.

A prominent problem in interacting with public technology is the visibility of the interaction to bystanders [7, 32]. Surprisingly, this continued to be the greatest issue in our study as well, whereas location tracking -related issues were much less prominent. This issue stems not only from the fact that bystanders are able to observe the users as they are interacting, but also that they are able to observe what kind of content they are interested in.

\section{CONCLUSION}

Public interactive systems can utilize location tracking in creative ways to enable new and seamless interaction scenarios. While such technologies have great potential in terms of both user experience and performance, they are not without potential pitfalls. For example, users may have specific requirements and preferences, and they may reject location-tracking systems in public altogether. Hence, we conducted a 24-participant user study wherein we explored the acceptance, perceptions, and concerns related to a public display system that tracks the location of users and personal mobile devices to create new interaction opportunities.

Our results suggest that users are largely accepting of, and excited about, novel interactive systems. However, such systems must provide clear value to users, be transparent about their ownership and use of data, and allow users to define preferences about when and where they are tracked. Moreover, the influence of the deployment setting is greatly emphasized when location-tracking features are added to an interactive display. Generally, users are more comfortable about location tracking in calm and/or professional contexts such as libraries, museums, office workspaces, and university campuses.

\section{REFERENCES}

[1] D. Anthony, T. Henderson, and D. Kotz. 2007. Privacy in Location-Aware Computing Environments. IEEE Pervasive Computing 6, 4 (Oct 2007), 64-72. https://doi.org/10.1109/MPRV.2007.83

[2] Carmelo Ardito, Paolo Buono, Maria Francesca Costabile, and Giuseppe Desolda. 2015. Interaction with Large Displays: A Survey. ACM Comput. Surv. 47, 3, Article 46 (Feb. 2015), 38 pages. https://doi.org/10.1145/2682623

[3] Louise Barkhuus. 2012. The Mismeasurement of Privacy: Using Contextual Integrity to Reconsider Privacy in HCI. In Proceedings of the SIGCHI Conference on Human Factors in Computing Systems (CHI '12). ACM, New York, NY, USA, 367-376. https://doi.org/10.1145/2207676.2207727

[4] Louise Barkhuus and Anind Dey. 2003. Is Context-Aware Computing Taking Control away from the User? Three Levels of Interactivity Examined. In UbiComp 2003: Ubiquitous Computing, Anind K. Dey, Albrecht Schmidt, and Joseph F. McCarthy (Eds.). Springer Berlin Heidelberg, Berlin, Heidelberg, 149-156. https: //doi.org/10.1007/978-3-540-39653-6_12

[5] Louise Barkuus and Anind Dey. 2003. Location-Based Services for Mobile Telephony: a Study of Users' Privacy Concerns. In Human-Computer Interaction INTERACT '03: IFIP TC13 International Conference on Human-Computer Interaction, 1st-5th September 2003, Zurich, Switzerland.

[6] Harry Brignull. 2011. Dark Patterns: Deception vs. Honesty in UI Design. Interaction Design, Usability 338 (2011). 
[7] Harry Brignull and Yvonne Rogers. 2003. Enticing People to Interact with Large Public Displays in Public Spaces. In Human-Computer Interaction INTERACT '03: IFIP TC13 International Conference on Human-Computer Interaction, 1st-5th September 2003, Zurich, Switzerland.

[8] Keith Cheverst, Alan Dix, Daniel Fitton, Chris Kray, Mark Rouncefield, Corina Sas, George Saslis-Lagoudakis, and Jennifer G. Sheridan. 2005. Exploring Bluetooth Based Mobile Phone Interaction with the Hermes Photo Display. In Proceedings of the 7th International Conference on Human Computer Interaction with Mobile Devices \&Amp; Services (MobileHCI '05). ACM, New York, NY, USA, 47-54. https: //doi.org/10.1145/1085777.1085786

[9] Elizabeth F Churchill, Les Nelson, Laurent Denoue, and Andreas Girgensohn. 2003. The plasma poster network: Posting multimedia content in public places.. In Human-Computer Interaction INTERACT '03: IFIP TC13 International Conference on Human-Computer Interaction, 1st-5th September 2003, Zurich, Switzerland, Vol. 3 599-606.

[10] Sarah Clinch, Nigel Davies, Thomas Kubitza, and Adrian Friday. 2014. Ownership and Trust in Cyber-Foraged Displays. In Proceedings of The International Symposium on Pervasive Displays (PerDis '14). ACM, New York, NY, USA, Article 168, 6 pages. https://doi.org/10.1145/2611009.2611010

[11] Sunny Consolvo, Ian E. Smith, Tara Matthews, Anthony LaMarca, Jason Tabert, and Pauline Powledge. 2005. Location Disclosure to Social Relations: Why, when, \& What People Want to Share. In Proceedings of the SIGCHI Conference on Human Factors in Computing Systems (CHI '05). ACM, New York, NY, USA 81-90. https://doi.org/10.1145/1054972.1054985

[12] Nigel Davies, Marc Langheinrich, Sarah Clinch, Ivan Elhart, Adrian Friday, Thomas Kubitza, and Bholanathsingh Surajbali. 2014. Personalisation and Privacy in Future Pervasive Display Networks. In Proceedings of the 32Nd Annual ACM Conference on Human Factors in Computing Systems (CHI '14). ACM, New York, NY, USA, 2357-2366. https://doi.org/10.1145/2556288.2557287

[13] Saul Greenberg, Sebastian Boring, Jo Vermeulen, and Jakub Dostal. 2014. Dark Patterns in Proxemic Interactions: A Critical Perspective. In Proceedings of the 2014 Conference on Designing Interactive Systems (DIS '14). ACM, New York, NY USA, 523-532. https://doi.org/10.1145/2598510.2598541

[14] Eva Hornecker and Matthias Stifter. 2006. Learning from Interactive Museum Installations About Interaction Design for Public Settings. In Proceedings of the 18th Australia Conference on Computer-Human Interaction: Design: Activities, Artefacts and Environments (OZCHI '06). ACM, New York, NY, USA, 135-142. https://doi.org/10.1145/1228175.1228201

[15] Hannu Kukka, Tommi Heikkinen, Henri Kytökangas, Taru Tanska, and Timo Ojala. 2018. UbiLibrary: Situated Large Public Display As Interactive Interface to Library Services. In Proceedings of the 22Nd International Academic Mindtrek Conference (Mindtrek '18). ACM, New York, NY, USA, 192-201. https://doi.org/ $10.1145 / 3275116.3275143$

[16] Marc Langheinrich. 2001. Privacy by Design - Principles of Privacy-Aware Ubiquitous Systems. In Proceedings of the 3rd international conference on Ubiquitous Computing. Springer, 273-291.

[17] Scott Lederer, Jennifer Mankoff, and Anind K. Dey. 2003. Who Wants to Know What when? Privacy Preference Determinants in Ubiquitous Computing. In $\mathrm{CHI}$ '03 Extended Abstracts on Human Factors in Computing Systems (CHI EA '03). ACM, New York, NY, USA, 724-725. https://doi.org/10.1145/765891.765952

[18] Bo Liu, Wanlei Zhou, Tianqing Zhu, Longxiang Gao, and Yong Xiang. 2018 Location Privacy and Its Applications: A Systematic Study. IEEE Access 6 (2018), 17606-17624. https://doi.org/10.1109/ACCESS.2018.2822260

[19] Joseph F. McCarthy, Ben Congleton, and F. Maxwell Harper. 2008. The Context, Content \& Community Collage: Sharing Personal Digital Media in the Physical Workplace. In Proceedings of the 2008 ACM Conference on Computer Supported Cooperative Work (CSCW '08). ACM, New York, NY, USA, 97-106. https://doi org/10.1145/1460563.1460580

[20] Alexander Meschtscherjakov, Wolfgang Reitberger, Michael Lankes, and Manfred Tscheligi. 2008. Enhanced Shopping: A Dynamic Map in a Retail Store. In Proceedings of the 10th International Conference on Ubiquitous Computing (UbiComp '08). ACM, New York, NY, USA, 336-339. https://doi.org/10.1145/1409635.1409680
[21] Jörg Müller, Florian Alt, Daniel Michelis, and Albrecht Schmidt. 2010. Requirements and Design Space for Interactive Public Displays. In Proceedings of the 18th ACM International Conference on Multimedia (MM '10). ACM, New York, NY, USA, 1285-1294. https://doi.org/10.1145/1873951.1874203

[22] Ville Mäkelä, Tomi Heimonen, and Markku Turunen. 2018. Semi-Automated, Large-Scale Evaluation of Public Displays. International fournal of $\mathrm{Hu}$ man-Computer Interaction 34, 6 (2018), 491-505. https://doi.org/10.1080/10447318. 2017.1367905

[23] Ville Mäkelä, Jobin James, Tuuli Keskinen, Jaakko Hakulinen, and Markku Turunen. 2017. "It's Natural to Grab and Pull": Retrieving Content from Large Displays Using Mid-Air Gestures. IEEE Pervasive Computing 16, 3 (2017), 70-77. https://doi.org/10.1109/MPRV.2017.2940966

[24] Ville Mäkelä, Mohamed Khamis, Lukas Mecke, Jobin James, Markku Turunen, and Florian Alt. 2018. Pocket Transfers: Interaction Techniques for Transferring Content from Situated Displays to Mobile Devices. In Proceedings of the 2018 CHI Conference on Human Factors in Computing Systems (CHI '18). ACM, New York, NY, USA, Article 135, 13 pages. https://doi.org/10.1145/3173574.3173709

[25] Ville Mäkelä, Sumita Sharma, Jaakko Hakulinen, Tomi Heimonen, and Markku Turunen. 2017. Challenges in Public Display Deployments: A Taxonomy of External Factors. In Proceedings of the 2017 CHI Conference on Human Factors in Computing Systems (CHI '17). ACM, New York, NY, USA, 3426-3475. https: //doi.org/10.1145/3025453.3025798

[26] T. Ojala, V. Kostakos, H. Kukka, T. Heikkinen, T. Linden, M. Jurmu, S. Hosio, F. Kruger, and D. Zanni. 2012. Multipurpose Interactive Public Displays in the Wild: Three Years Later. Computer 45, 5 (May 2012), 42-49. https://doi.org/10.1109/ MC. 2012.115

[27] T. Ojala, H. Kukka, T. Lindén, T. Heikkinen, M. Jurmu, S. Hosio, and F. Kruger. 2010. UBI-Hotspot 1.0: Large-Scale Long-Term Deployment of Interactive Public Displays in a City Center. In 2010 Fifth International Conference on Internet and Web Applications and Services. 285-294. https://doi.org/10.1109/ICIW.2010.49

[28] Peter Peltonen, Esko Kurvinen, Antti Salovaara, Giulio Jacucci, Tommi Ilmonen, John Evans, Antti Oulasvirta, and Petri Saarikko. 2008. It's Mine, Don'T Touch!: Interactions at a Large Multi-touch Display in a City Centre. In Proceedings of the SIGCHI Conference on Human Factors in Computing Systems (CHI '08). ACM, New York, NY, USA, 1285-1294. https://doi.org/10.1145/1357054.1357255

[29] Mahsan Rofouei, Andrew Wilson, A.J. Brush, and Stewart Tansley. 2012. Your Phone or Mine?: Fusing Body, Touch and Device Sensing for Multi-user Devicedisplay Interaction. In Proceedings of the SIGCHI Conference on Human Factors in Computing Systems (CHI '12). ACM, New York, NY, USA, 1915-1918. https: //doi.org/10.1145/2207676.2208332

[30] Albrecht Schmidt, Bastian Pfleging, Florian Alt, Alireza Sahami, and Geraldine Fitzpatrick. 2012. Interacting with 21st-Century Computers. IEEE Pervasive Computing 11, 1 (January 2012), 22-31. https://doi.org/10.1109/MPRV.2011.81

[31] Fabius Steinberger, Marcus Foth, and Florian Alt. 2014. Vote With Your Feet: Local Community Polling on Urban Screens. In Proceedings of The International Symposium on Pervasive Displays (PerDis '14). ACM, New York, NY, USA, Article 44, 6 pages. https://doi.org/10.1145/2611009.2611015

[32] Nina Valkanova, Robert Walter, Andrew Vande Moere, and Jörg Müller. 2014. MyPosition: Sparking Civic Discourse by a Public Interactive Poll Visualization. In Proceedings of the 17th ACM Conference on Computer Supported Cooperative Work \&\#38; Social Computing (CSCW'14). ACM, New York, NY, USA, 1323-1332. https://doi.org/10.1145/2531602.2531639

[33] Roy Want, Andy Hopper, Veronica Falcão, and Jonathan Gibbons. 1992. The Active Badge Location System. ACM Trans. Inf. Syst. 10, 1 (Jan. 1992), 91-102. https://doi.org/10.1145/128756.128759

[34] Andrew D. Wilson and Hrvoje Benko. 2014. CrossMotion: Fusing Device and Image Motion for User Identification, Tracking and Device Association. In Proceedings of the 16th International Conference on Multimodal Interaction (ICMI '14). ACM, New York, NY, USA, 216-223. https://doi.org/10.1145/2663204.2663270

[35] Chi-Jui Wu, Steven Houben, and Nicolai Marquardt. 2017. EagleSense: Tracking People and Devices in Interactive Spaces Using Real-Time Top-View DepthSensing. In Proceedings of the 2017 CHI Conference on Human Factors in Computing Systems (CHI '17). ACM, New York, NY, USA, 3929-3942. https://doi.org/10. $1145 / 3025453.3025562$ 